\title{
Ion channels PIEZOs identified as the long-sought baroreceptor mechanosensors for blood pressure control, and other updates on autonomic research
}

\author{
Mitchell G. Miglis ${ }^{1} \cdot$ Srikanth Muppidi ${ }^{1}$
}

Received: 24 December 2018 / Accepted: 27 December 2018 / Published online: 2 January 2019

(c) Springer-Verlag GmbH Germany, part of Springer Nature 2019

Keywords Baroreflex $\cdot$ Autonomic failure $\cdot$ Orthostatic hypotension $\cdot$ Atomoxetine $\cdot$ Pyridostigmine

\section{Pyridostigmine and atomoxetine: a whole greater than the sum of their parts}

The treatment of neurogenic orthostatic hypotension $(\mathrm{nOH})$ in patients with sympathetic failure is a challenging task for autonomic clinicians. Blood pressure (BP) can be erratic, with hypotension and syncope, as well as supine hypertension and its consequences. Current pharmacological options for $\mathrm{nOH}$ are limited, especially in patients with severe supine hypertension, and the efficacy of the available medications is inconsistent. Therefore, the development of new therapeutic options is always of interest.

Okamoto et al. [1] at Vanderbilt University have recently described the synergistic effect of atomoxetine and pyridostigmine for the treatment of $\mathrm{nOH}$ in a mixed group of patients with autonomic failure. Neither atomoxetine nor pyridostigmine are new medications, having been on the market for decades (pyridostigmine was patented in 1945). Atomoxetine, currently approved by the US Food and Drug Administration for the treatment of attention deficit hyperactivity disorder, is a norepinephrine transporter (NET) blocker, thus preventing the reuptake of norepinephrine in the brain and peripheral autonomic nerves. Small, shortterm studies have shown the promising effects of atomoxetine for the treatment of $\mathrm{nOH}$ [2]. It also inhibits the reuptake of dopamine in specific brain regions such as the prefrontal cortex, which along with increased central norepinephrine, results in its stimulant effects. Pyridostigmine,

Srikanth Muppidi

muppidis@stanford.edu

1 Stanford Medical Center, Stanford Neurosciences Health Center, 213 Quarry Road, 2nd Floor, Palo Alto, CA 94304, USA an anti-cholinesterase inhibitor commonly prescribed for the treatment of myasthenia gravis, is sometimes used for the treatment of nOH due to its presumed mechanism of enhancing acetylcholine neurotransmission at the level of the sympathetic ganglia [3, 4]. Okamoto and colleagues thus hypothesized that the combination of atomoxetine and pyridostigmine would result in a synergistic pressor effect by increasing residual sympathetic tone, even though not all patients always respond to these treatments. For instance, atomoxetine appears to be more effective in patients with multiple system atrophy (MSA), in whom the peripheral sympathetic innervation is preserved, as compared to patients with pure autonomic failure (PAF), in whom sympathetic innervation and norepinephrine production is severely impaired [5].

Okamoto and colleagues enrolled a total of 12 patients with $\mathrm{nOH}$ and severe autonomic failure (five with PAF, three with probable MSA, two with Parkinson's disease, one with amyloidosis, and one with autonomic failure of unknown cause) after careful screening and diagnosis based on clinical evaluation and autonomic testing (Valsalva maneuver, heart rate during deep breathing, hyperventilation, cold pressor and handgrip testing, and a 10-min orthostatic stand test). The neurogenic nature of $\mathrm{OH}$ was defined by the authors as an "abnormal response to Valsalva maneuver." All patients had measurements of supine and standing plasma catecholamines. Patients were asked to stand $10 \mathrm{~min}$ or as long as they could tolerate. After sitting again they were given a single dose of study drug: a single dose of placebo, $18 \mathrm{mg}$ of atomoxetine, $60 \mathrm{mg}$ of pyridostigmine, or the combination of atomoxetine and pyridostigmine, in a single-blind, crossover fashion. BP and heart rate (HR) were measured for the following hour, and the 10-min stand test was then repeated. Medication trials with placebo, atomoxetine, and pyridostigmine were done on separate days in a random 
order, either on consecutive days or a day apart. Patients were asked to rate the severity of their orthostatic symptoms immediately after their orthostatic stress test using the Orthostatic Hypotension Symptom Assessment (OHSA) score [6]. The primary outcome was seated systolic BP during the 60 -min post-drug period. The OHSA score were the secondary outcome measure.

The mean orthostatic drop in systolic BP was $62 \pm 7 \mathrm{mmHg}$. Consistent with the authors' hypothesis, the combination of atomoxetine and pyridostigmine significantly increased seated SBP compared to placebo and each drug alone, with the maximal increase $(+33 \pm 8 \mathrm{mmHg})$ seen 60-min after the administration of both drugs. The change in the difference from baseline to 1-min standing $\mathrm{BP}$ (systolic/diastolic) was $-2 \pm 4 / 1 \pm 5 \mathrm{mmHg}$ with placebo, $14 \pm 6 / 6 \pm 5 \mathrm{mmHg}$ with atomoxetine, $-1 \pm 5 / 1 \pm 4 \mathrm{mmHg}$ with pyridostigmine, and $+20 \pm 9 / 10 \pm 3 \mathrm{mmHg}$ with the combination. The range of BP responses to atomoxetine, however, was wide with significant overlap between groups, suggesting that the pressor effect mainly depends on the presence of residual sympathetic tone with norepinephrine release from peripheral sympathetic nerves, although there was no relevant difference by diagnosis type (i.e. PAF vs. MSA). Only the combination of medications showed an improvement in orthostatic tolerance as measured by the OHSA $(19.9 \pm 5.0$ with combination vs. $29.2 \pm 4.5$ with placebo; $P=0.023$ ); however, only eight patients completed orthostatic symptom scores for all treatment arms. No significant correlation was found between supine or upright plasma norepinephrine levels and the seated or upright BP.

Potential limitations of this study include a small sample size, with a power calculation based on orthostatic BP responses to only three patients (there is significant variability in BP responses across disease states, and even within the same patient). The authors did not monitor BP beyond $1 \mathrm{~h}$ after drug administration. Both atomoxetine and pyridostigmine have durations of action that extend beyond $1 \mathrm{~h}$, thus the long-term effect on BP and risk of supine hypertension was not measured. In fact, the authors did not measure supine $\mathrm{BP}$, which is a significant limitation.

Nonetheless, this small but impactful study provides the autonomic community with important data on a new potential drug combination in the treatment of autonomic failure. It demonstrates several clinically useful points: (1) the pressor effect of pyridostigmine by itself is very limited, (2) both atomoxetine and pyridostigmine are well tolerated, and (3) the combination of these two medication, by nature of their synergistic pharmacologic effect, produce a whole that is significantly greater than their respective parts.

\section{How does mechanical stretch translate to neuronal signaling for optimal baroreflex function?}

The baroreceptors, stretch receptors located within the walls of the carotid sinus and aortic arch, are essential for continuous BP and heart rate regulation. Failure in conveying the afferent signals from baroreceptors (i.e., baroreflex failure caused by acquired lesions or rare genetic mutations) can result in hypertension, excessive BP variability, orthostatic hypotension and cardiac arrhythmias. While the importance of baroreflex function has been well known for decades, the exact nature of these mechanoreceptors has not been fully elucidated. Multiple receptors including TRCP5, TRPV1, and ASIC2 have been described. However, inhibition of these with genetic models and pharmacological techniques did not always lead to convincing results, raising the possibility of other unknown factors.

Zeng and colleagues, in their paper PIEZOs mediate neuronal sensing of blood pressure and the baroreceptor reflex published in a recent issue of Science, identified the mechanically activated ion channels PIEZO1 and PIEZO2 as the key receptors involved in baroreceptor function [7]. PIEZO1 is prominently expressed in the cardiovascular system and PIEZO2 is seen in various sensory neurons. To prove their hypothesis, the authors performed multiple different in vivo tests while monitoring BP and heart rate. They labeled carotid sinus neurons and found both PIEZO1- and PIEZO2-positive receptors, then tested the effect of various medications in the PIEZO1 knockout, PIEZO2 knockout, and double knockout (DKO) mice. They noticed that phenylephrine-induced bradycardia was absent and baroreceptor sensitivity was eradicated in DKO mice, but not in PIEZO1 or PIEZO2 mice. Phenylephrineinduced hypertension was also significantly higher in DKO mice. Similarly, changes in heart rate after sodium nitroprusside were reduced in DKO mice. They also tested BP variability in freely moving conscious mice. DKO mice had higher mean arterial pressure during activity and a higher BP variability when compared to wild-type mice. Additionally, spontaneous baroreflex sensitivity testing was severely reduced in DKO mice. DKO mice also had higher homovanillic acid (a major catecholamine metabolite) levels compared to wild-type mice, likely a reflection of higher catecholamine production.

These experiments support the hypothesis that functional loss of PIEZO receptors results in impaired baroreceptor function, and that PIEZO1 and PIEZO2 receptors together are critical mechanoreceptors in baroreflex function. It is likely that there are other mechanical or sensory receptors factors beyond PIEZO receptors, however this knowledge has the potential to impact clinical practice if 
medications could be designed to target these receptors. It is unclear, however, if potential side effects would limit drug development (e.g., PIEZO mutations are known to cause congenital arthrogryposis) [8,9]. Regardless of whether this discovery will translate to new therapies in the short term, we finally came to understand how a pure mechanical change or pressure change is translated to neuronal signaling in one of the most essential reflex mechanisms of the human body.

Funding support None.

\section{Compliance with ethical standards}

Conflict of interest None.

\section{References}

1. Okamoto LE, Shibao CA, Gamboa A, Diedrich A, Raj SR, Black BK, Robertson D, Biaggioni I (2019) Synergistic pressor effect of atomoxetine and pyridostigmine in patients with neurogenic orthostatic hypotension. Hypertension 73:235-241

2. Ramirez CE, Okamoto LE, Arnold AC, Gamboa A, Diedrich A, Choi L, Raj SR, Robertson D, Biaggioni I, Shibao CA (2014)
Efficacy of atomoxetine versus midodrine for the treatment of orthostatic hypotension in autonomic failure. Hypertension 64:1235-1240

3. Sandroni P, Opfer-Gehrking TL, Singer W, Low PA (2005) Pyridostigmine for treatment of neurogenic orthostatic hypotension [correction of hypertension] - a follow-up survey study. Clin Auton Res 15:51-53

4. Singer W, Sandroni P, Opfer-Gehrking TL, Suarez GA, Klein CM, Hines S, O'Brien PC, Slezak J, Low PA (2006) Pyridostigmine treatment trial in neurogenic orthostatic hypotension. Arch Neurol 63:513-518

5. Shibao C, Martinez J, Palma JA, Kaufmann H, Biaggioni I (2017) Norepinephrine levels predicts the improvement in orthostatic symptoms after atomoxetine in patients with neurogenic orthostatic hypotension. Neurology 88:P5.320

6. Kaufmann H, Malamut R, Norcliffe-Kaufmann L, Rosa K, Freeman R (2012) The Orthostatic Hypotension Questionnaire (OHQ): validation of a novel symptom assessment scale. Clin Auton Res 22:79-90

7. Zeng WZ, Marshall KL, Min S, Daou I, Chapleau MW, Abboud FM, Liberles SD, Patapoutian A (2018) PIEZOs mediate neuronal sensing of blood pressure and the baroreceptor reflex. Science 362:464-467

8. Parpaite T, Coste B (2017) Piezo channels. Curr Biol CB 27:R250-R252

9. Wu J, Lewis AH, Grandl J (2017) Touch, tension, and transduction-the function and regulation of piezo ion channels. Trends Biochem Sci 42:57-71 